Communications in Physics, Vol.27, No. 1 (2017), pp. 37-43

DOI:10.15625/0868-3166/27/1/8886

\title{
ELECTROCHEMICAL FABRICATION OF HYBRID PLASMONIC-DIELECTRIC NANOMATERIALS BASED ON GOLD-DIAMOND CLUSTERS
}

\author{
DO HOANG TUNG ${ }^{1}$, TRAN THI THUONG ${ }^{1}$, NGUYEN THANH LIEM ${ }^{1}$, \\ PHAM VAN DUONG ${ }^{1}$, NGHIEM THI HA LIEN ${ }^{1}$, PHAM HONG MINH ${ }^{1, \dagger}$, \\ ANDREY ALEKSEEVICH IONIN ${ }^{2}$, ALEXEY OLEGOVICH LEVCHENKO ${ }^{2}$, \\ ANDREY ANDREEVICH RUDENKO ${ }^{2}$, IRINA NIKOLAEVNA SARAEVA ${ }^{2}$, \\ ANASTASIYA KONSTANTINOVNA IVANOVA ${ }^{2,3}$, \\ SERGEY IVANOVICH KUDRYASHOV ${ }^{2,3}$, \\ NGUYEN LUONG ${ }^{2,4}$ AND NGUYEN THI HUYEN TRANG ${ }^{2,4}$ \\ ${ }^{1}$ Institute of Physics, Vietnam Academy of Science and Technology, \\ 18 Hoang Quoc Viet, Cau Giay, Hanoi, Vietnam \\ ${ }^{2}$ Lebedev Physical Institute, Leninskiy prospect 53, 119991 Moscow, Russia \\ ${ }^{3}$ National Research Nuclear University MEPhI (Moscow Engineering Physics Institute), \\ Kashirskoeshosse 31, 115409 Moscow, Russia \\ ${ }^{4}$ Moscow Institute of Physics and Technology MIPT (National University), Institutsky Lane 9, \\ 141701 Dolgoprudny, Russia \\ $\dagger$ E-mail: phminh@iop.vast.ac.vn
}

Received 25 November 2016

Accepted for publication 26 September 2017

\begin{abstract}
Hybrid plasmonic-dielectric materials were fabricated by micro-discharge through water sols of sub-micrometer-sized diamonds mixed with $\mathrm{HAuCl}_{4}$ acid. Primary characterization of their deposits on a silicon wafer surface by means of electron microscopy and energy-dispersive $x$-ray spectroscopy indicate close proximity of gold nanoparticles and diamond particles, which is supported by photoluminescence studies demonstrating strong - almost two-fold - damping of diamond luminescence owing to the attachment of gold nanoparticles. UV-near IR spectroscopy of their sols consistently exhibits small red spectral shifts for the fabricated nanomaterials, comparing to bare gold nanoparticles.
\end{abstract}

Keywords: micro-diamonds, gold nanoparticles, hybrid plasmonic-dielectric materials, electrochemical fabrication, electron microscopy and optical characterization..

Classification numbers: 52.50.Jm, 87.85.Rs.

(C)2017 Vietnam Academy of Science and Technology 


\section{INTRODUCTION}

Novel hybrid combination of plasmonic-resonant metallic and polaritonic-resonant dielectric or semiconductor nanoparticles was recently proposed [1-4] and demonstrated both as advanced nanomaterial and meta-surfaces [5]. Currently, two main structural designs of such metaldielectric (hybrid) nanoparticles were proposed. In the first case, a cluster of closely arranged two or few metallic and dielectric nanoparticles can be produced by colloidal chemistry, laser printing (laser-induced forward transfer) or nano-lithography [1,2]. Alternatively, nanoparticles embedded one into another ("core-shell") were produced as "dielectric core-metallic shell" [3] or "semiconductor core-metallic shell" [4] features. Meanwhile, there is also an intermediate case of a dielectric or semiconductor particle doped by metallic nano-impurities [6]. Later on, such structures became a subject of broad review [7]. Hence, simple, high-yield and selective methods for fabrication of such and other possible promising optical materials are demanding to enable their future broader investigation.

In this work, electrochemical reduction of $\mathrm{HAuCl}_{4}$ acid in dense hydrosols of high-index diamond sub-micrometer sized particles was tested as a promising "green" fabrication way of "metallic nanoparticle-diamond particle" clusters in their colloidal solutions. Plasma-enabled approach requires a only water-based solution with the metal precursor and completely free of any added reducing and/or capping agents. This synthesis technique results in a largely simple, rapid and clean method that can greatly facilitate the subsequent surface functionalization of AuNPs [8].

The produced hybrid species were preliminarily characterized in terms of their structure and chemical composition, electronic, optical and plasmonics properties, being considered as a novel potential optical nano-platform for chemo and bio-sensing in future.

\section{EXPERIMENTAL}

Sub-micron (diameter $<1 \mu \mathrm{m}$ ) diamond (D) particles were used in our studies, being separated through centrifugation upon milling of plasma-enhanced chemically vapor deposited (PECVD) diamonds. Gold nanoparticles (Au NPs) were produced by micro-discharge through a $0.05 \mathrm{mM} / 1 \mathrm{HAuCl}_{4}$ solution in deionized water (Fig. 1). Au(III) chloride trihydrate (99.9\%; $\mathrm{HAuCl}_{4} 3 \mathrm{H}_{2} \mathrm{O}$ ) was purchased from Sigma-Aldrich.

The micro-plasma system for gold reduction consists of a DC high voltage source, a plasma nozzle, a gold electrode and a 10-ml glass beaker from Bomex with $6 \mathrm{ml}$ of the precursor solution. The plasma nozzle has a teflon housing with one end being a hollow teflon cylinder with outer diameter of $15 \mathrm{~mm}$ and inner diameter of $10 \mathrm{~mm}$, covering the plasma electrode, and the other end being a 6-mm quick connector. The plasma electrode is a 1-mm diameter tungsten rod connected to the negative polarity via a $100-\mathrm{k} \Omega$ resistor. The other polarity is grounded and connected to the gold electrode, which is submerged into the precursor solution. The processing current is maintained constant $(5 \mathrm{~mA})$. The temperature of the $6 \mathrm{ml}$ water solution exposed to the plasmasustained at $5 \mathrm{~mA}$ current for $5 \mathrm{~min}$, was observed to increase from about 25 to about $40^{\circ} \mathrm{C}$.

The produced red-blue Au nanoparticle sols were characterized by a scanning electron microscope JSM 7001F (SEM, JEOL), supplied by a energy-dispersive X-ray spectroscopy (EDX) module INCA (Oxford Instruments), in terms of size distributions as their deposits, dried on atomically smooth silicon wafer substrates. Their optical densities were measured over the range of $300-800 \mathrm{~nm}$, including the interband transition $(380-390 \mathrm{~nm})$ and plasmon resonance $(\sim 520-$ 
$530 \mathrm{~nm}$ ) bands [9], in a 1-cm wide cuvette by means of a UV-near IR spectrometer V-570 (Jasco). Photoluminescence studies of the colloidal solutions were performed by means of a spectrofluorimeterFluoromax-4 (Horiba Jobin-Yvon) at the $360 \mathrm{~nm}, 400 \mathrm{~nm}$ and $480 \mathrm{~nm}$ excitation wavelengths.

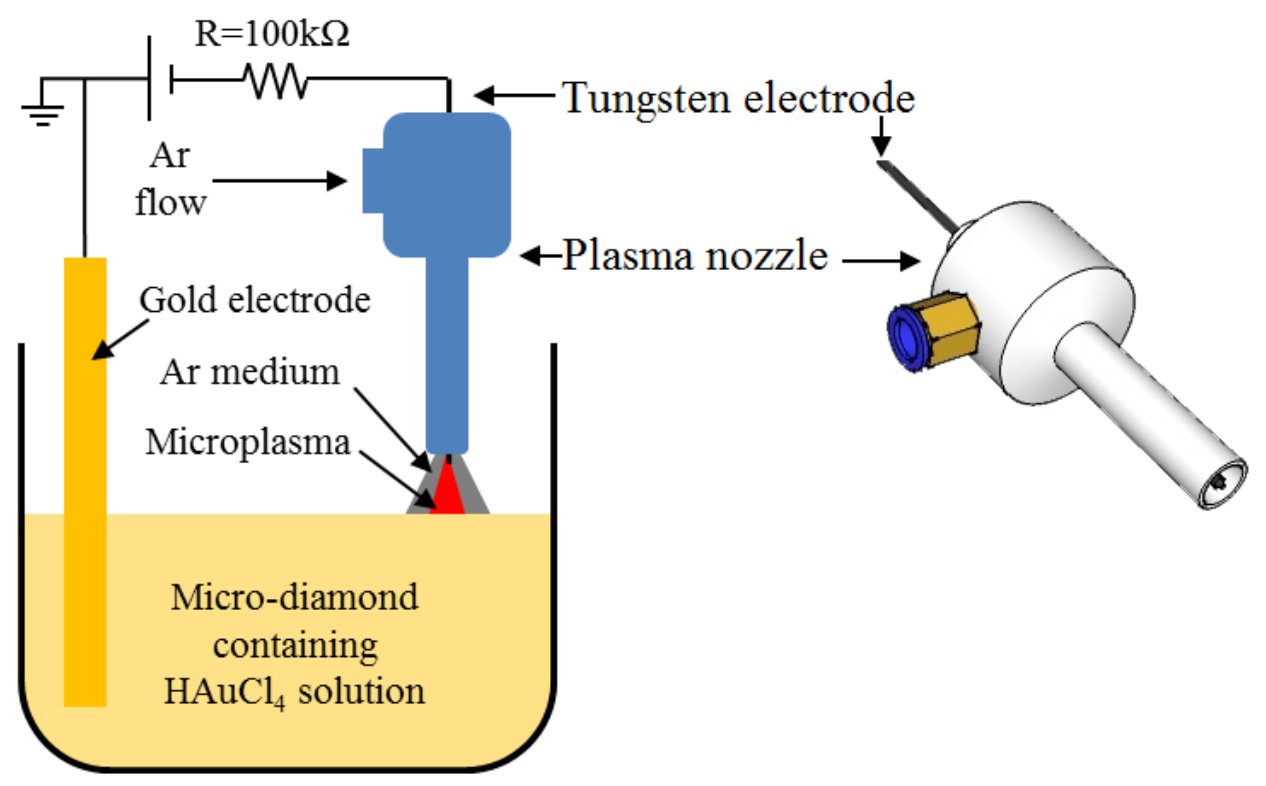

Fig. 1. Micro-discharge work-station for electrochemical gold reduction.

\section{RESULTS AND DISCUSSION}

SEM studies of the produced Au-NP electrochemically-decorated D-particles indicate the nanoscale proximity of the metallic nano- and dielectric sub-micrometer species, with considerable surface coverage for D-particles (Fig. 2c). This was also identified by means of highresolution EDX spectroscopy (Figs. 2(d)-(f)).

Indeed, the surrounding Au nanoparticles significantly influence the measured yield of photoluminescence intensity from non- and decorated diamond particles, reducing it by $50 \%$ for the 400-nm excitation (Fig. 3, left). More detailed comparison performed for the photoexcitation at $480 \mathrm{~nm}$, indicates that the mechanical mixture of Au-NP and D-particle colloids, preserving the concentration of D-particles, results only in 30\% reduction of PL intensity, while the decorated D-particles exhibit corresponding 60\% reduction. Such substantial damping of PL intensity indicates direct electrical contact between the diamond and metallic species, resulting in non-radiative sink of photo-excited carriers via their tunneling into the metallic nanoparticles [10,11].

Then, the corresponding measurements of optical density, including in this case both absorption and scattering/diffuse transmission (extinction), demonstrate distinct light scattering on 
non- and decorated D-particles in sols, monotonously decreasing for UV to IR range, a diffuse band of interband transitions $(<500 \mathrm{~nm})$ and a distinct dipolar surface plasmon resonance $(\approx$ $520 \mathrm{~nm}$ ) in gold nanoparticles [9] - both bare and attached ones (Fig. 4). In the presence of the D-particles, substantial "red" spectral shifts of the plasmonic resonance are observed for the gold nanoparticles (Fig. 4), being more pronounced for the decorated diamond particles $(\approx 536 \mathrm{~nm})$, than for those adsorbing Au nanoparticles on their surfaces from the mechanical mixture of their colloids $(\approx 530 \mathrm{~nm})$. These shifts of the plasmon resonance peak can be unambiguously related to the high-index dielectric environment of the Au nanoparticles, provided by their attachment to the diamonds [12], provided by the diamond particles to the metallic nanoparticles.
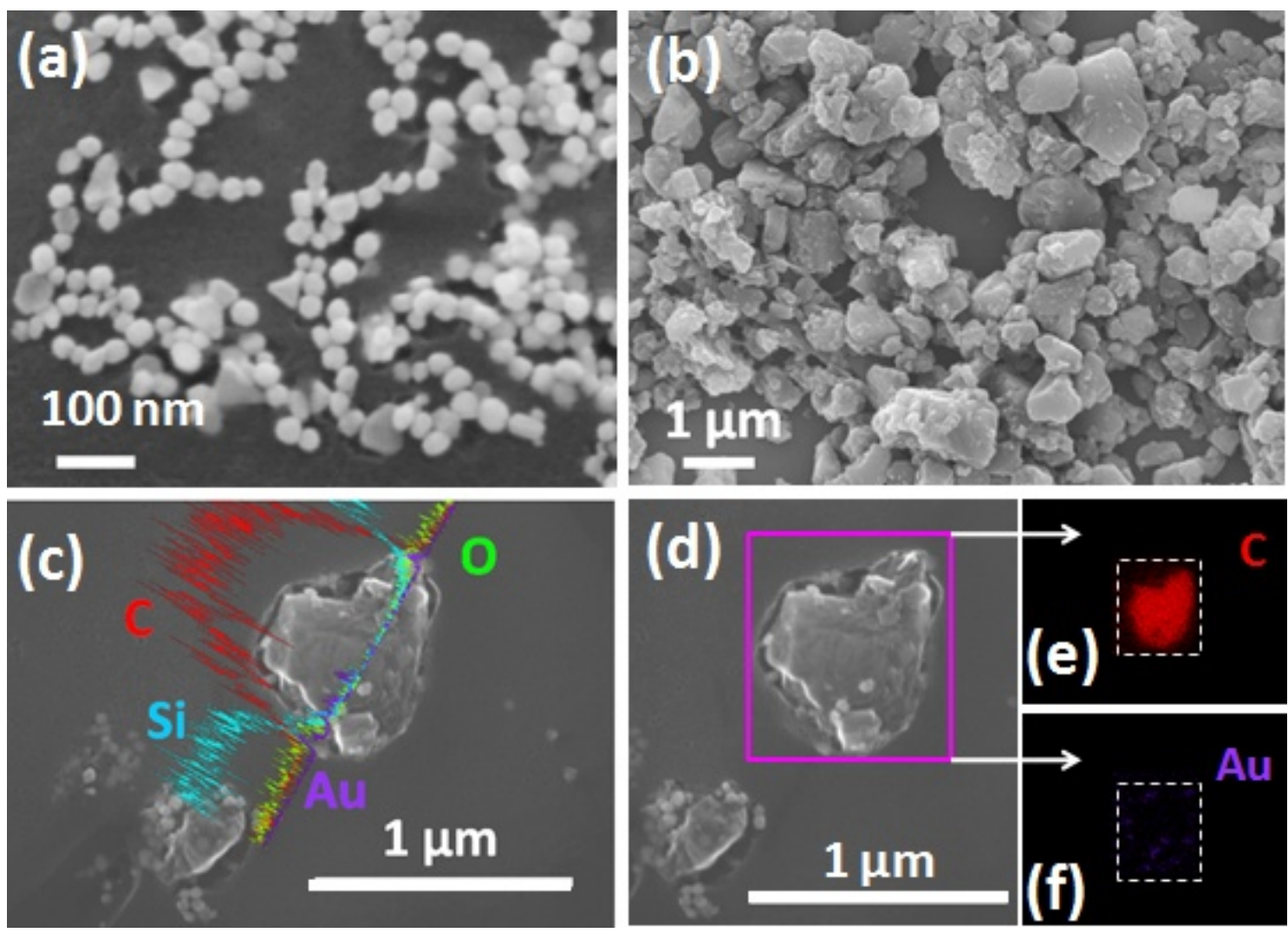

Fig. 2. Magnified top-view SEM images of electrochemically produced gold nanoparticles (a), initial diamond particles (b), EDX-profile of the decorated D-particle deposited on the Si-wafer surface (c) and its corresponding elemental (carbon $-\mathrm{C}$, gold $-\mathrm{Au}$ ) maps (d,e,f). 


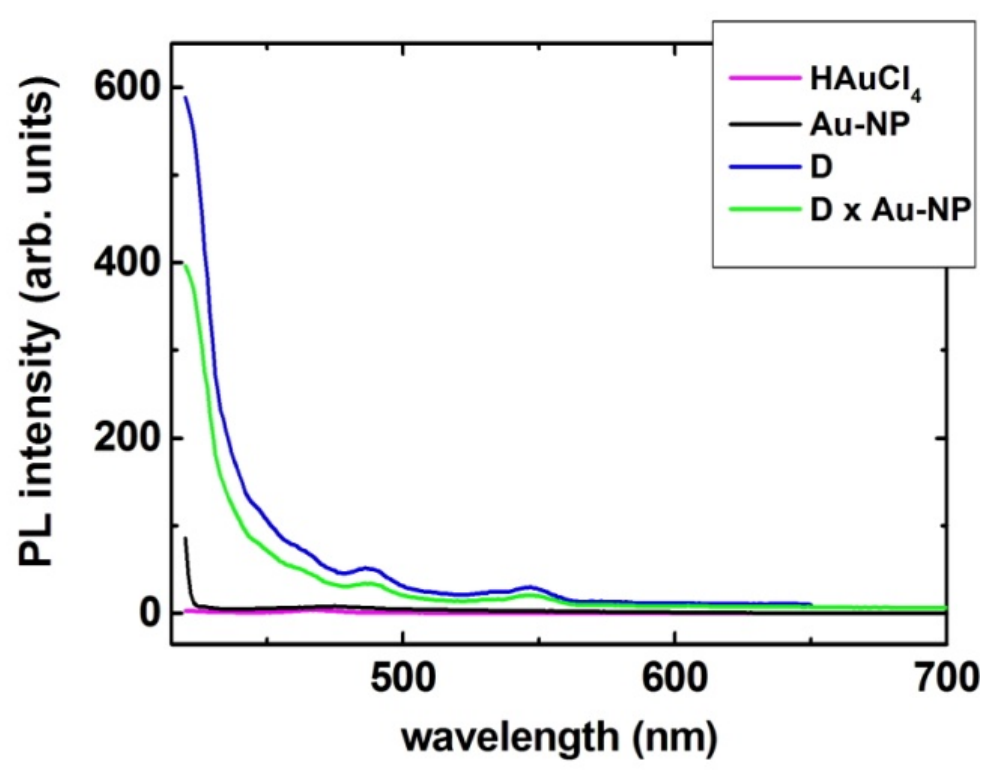

(a)

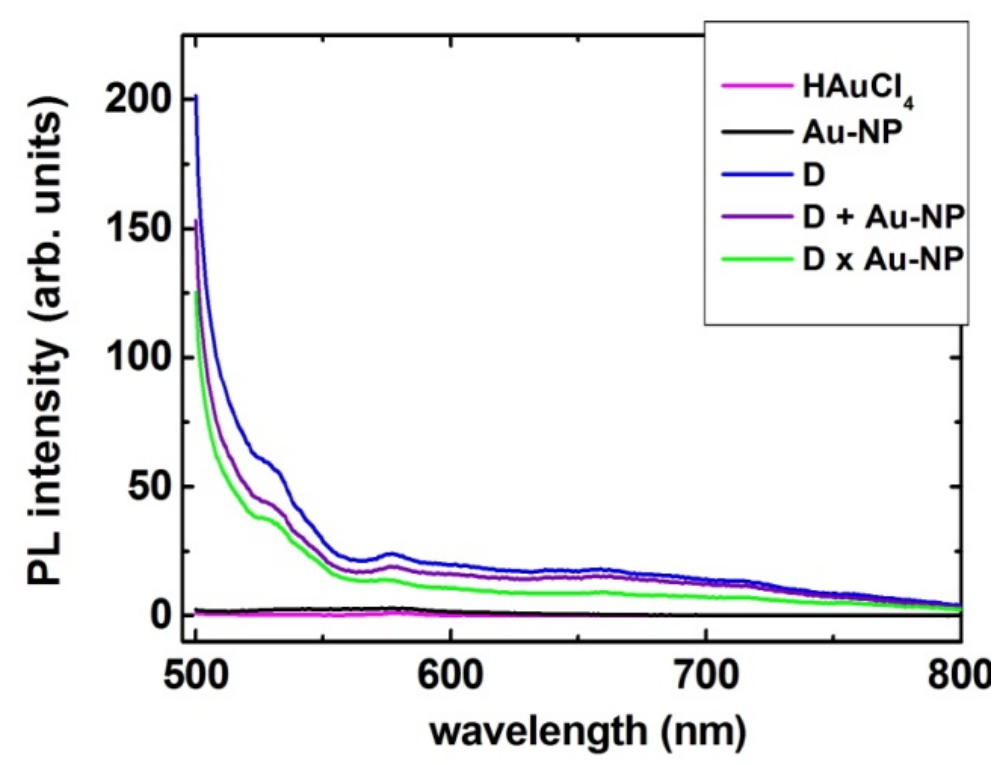

(b)

Fig. 3. Comparative photoluminescence spectra of the precursor $\mathrm{HAuCl}_{4}$ solution $\left(\mathrm{HAuCl}_{4}\right)$, bare Au-nanoparticles (Au-NP), bare diamond particles (D), diamond particles mixed with (D + Au-NP) or decorated by Au-nanoparticles (D x Au-NP), all - in water sols. PL luminescence was excited at $400 \mathrm{~nm}$ (a) and $480 \mathrm{~nm}$ (b). 


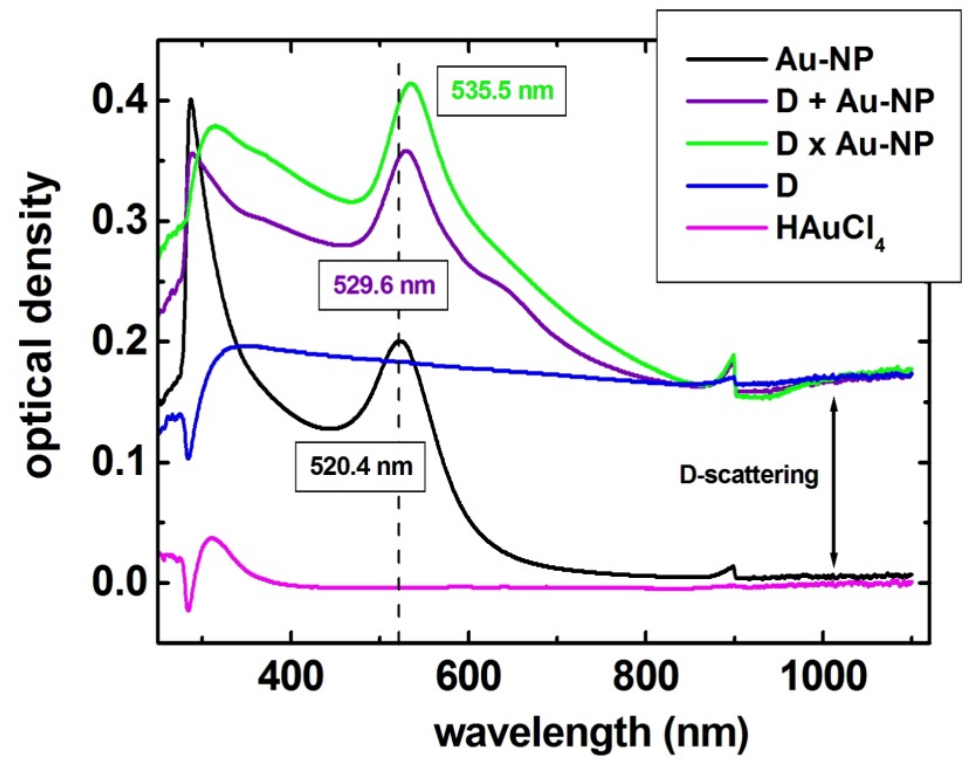

Fig. 4. Extinction UV-near-IR spectra of bare Au-nanoparticles, diamond particles mixed with or decorated by Au-nanoparticles, bare diamond particles in water sols and the precursor $\mathrm{HAuCl}_{4}$ solution. The color marks show the corresponding positions of the surface plasmon resonance for the Au-nanoparticles in the different ambient, while the bilateral arrow indicates the scattering level of the diamond particles in water sols.

\section{CONCLUSION}

Hybrid plasmonics-dielectric nanomaterials were fabricated by micro-discharge through the water sols of sub-micrometer sized diamond particles mixed with $\mathrm{HAuCl}_{4}$ acid. Their preliminary electron microscopy and energy-dispersive x-ray spectroscopy characterization indicates the near proximity of gold nanoparticles with nanodiamonds, which is strengthened till their direct binding.Photoluminescence studies show strong - 50-60\% - damping of diamond luminescence owing to the attachment of gold nanoparticles.UV-near IR optical density measurements exhibit a small red spectral shift for the electrochemically-fabricated nanomaterials, indicating the dielectric medium effect of diamond on plasmonic characteristics of the attached Au nanoparticles.

\section{ACKNOWLEDGEMENTS}

This research was supported by the collaborative grant VAST.HTQT.NGA.11/16-17and the Russian Foundation for Basic research (nos. 16-52-540002 Viet-a, 15-52-04037 Bel_mol_a).

This work was also supported in part by the Competitiveness Program of NRNU MEPhI.

\section{REFERENCES}

[1] L. Shi, T. U. Tuzer, R. Fenollosa and F. Meseguer, Adv. Mater. 24 (2012) 5934.

[2] U. Zywietz, A. B. Evlyukhin, C. Reinhardt and B. N. Chichkov, Nature Comm. 5 (2014) 3402.

[3] J. Kundu, F. Le, P. Nordlander and N. J. Halas, Chem. Phys. Lett. 452 (2008) 115. 
[4] D. A. Zuev, S. V. Makarov, V. A. Milichko, S. V. Starikov, I. S. Mukhin, I. A. Morozov, I. I. Shishkin, A. E. Krasnok and P. A. Belov, Adv. Mater. 28 (2016) 3087.

[5] R. Jiang, B. Li, C. Fang and J. Wang, Adv. Mater. 26 (2014) 52745309.

[6] A. B. Evlyukhin, A. L.Stepanov,, A. V.Dmitriev, A. S. Akhmanov, V. N. Bagratashvili and B. N. Chichkov, Opt. Comm. 316 (2014) 56.

[7] D.Smirnova, Y. S Kivshar, Multipolar nonlinear nanophotonics, arXiv preprint arXiv:1609.02057.

[8] J. Patel, L. Nemcova, P. Maguire, W. G. Grahamand, D. Mariotti, Nanotechnology 24 (2013) 245604.

[9] V. Amendola, M. Meneghetti, J. Phys. Chem. C 113 (2009) 4277.

[10] A Rajan, G Kaur, A Paliwal, H K Yadav, V Gupta and M Tomar, J. Phys. D: Appl. Phys. 47 (2014) 425102.

[11] H. Fan, M. Sun, P. Ma, Min Yin, Linfeng Lu, X.Xue, X. Zhu, D. Li and J. Ma, Nanotechnology 27 (2016) 365303.

[12] V. Myroshnychenko, J. Rodríguez-Fernández, I. Pastoriza-Santos, A. M. Funston, C. Novo, P. Mulvaney, L. M. Liz-Marzán and F. J. G. de Abajo, Chem. Soc. Rev. 37 (2008) 1792. 